\title{
Tumor fibroso solitario de la pleura asociado a síndromes paraneoplásicos*
}

\author{
Drs. FELIPE LEÓN F. ${ }^{1}$, FELIPE BANNURA Y. ${ }^{1}$, MARÍA E. SOLOVERA R. ${ }^{1}$, PATRICIO SALAS P. ${ }^{1}$ \\ 1 Departamento de Cirugía de Tórax. Pontificia Universidad Católica de Chile. \\ Santiago, Chile.
}

\begin{abstract}
Solitary fibrous tumor of pleura associated with paraneoplastic syndromes

Introduction: Solitary fibrous tumors of pleura (SFTP) are occasionally associated with paraneoplastic syndromes such as hypoglycemia and diffuse skeletal hyperostosis. Clinical case: We report a 60 years old male presenting with episodes of disorientation and inappropriate behavior, along with cough and weight loss. On physical examination there was digital clubbing. A chest $\mathrm{X}$ ray showed a big tumor in the right hemithorax. Fasting blood glucose was $24 \mathrm{mg} / \mathrm{dl}$ and erythrocyte sedimentation rate was elevated. Large bone $\mathrm{X}$ rays showed hyperostosis. A needle biopsy of the tumor showed a SFTP. The tumor was excised and the final pathological diagnosis was a benign SFTP. Hypoglycemia subsided after surgery. After four months of follow up, finger clubbing was disappearing.
\end{abstract}

Key words: Solitary fibrous tumor of pleura, hypoglycemia, hyperostosis.

\section{Resumen}

Introducción: Los Tumores Fibrosos Solitarios de la Pleura (TFSP) son neoplasias infrecuentes, consideradas lesiones benignas en general, con potencial maligno dependiendo de su histología. Se asocian en raras ocasiones a síndromes paraneoplásicos como la hipoglicemia (Síndrome de Doege-Potter) e hiperostosis ósea difusa (osteoartropatía hipertrófica pulmonar). Caso clínico: Consulta un varón de 60 años en el Hospital Clínico de la Pontificia Universidad Católica de Chile por episodios de desorientación y conductas inapropiadas, junto con tos y baja de peso. Además refiere dolor en extremidades y aumento de volumen de falanges distales de manos. Al examen destaca disminución del murmullo pulmonar a derecha e hipocratismo digital. Se estudia con radiografía y tomografía de tórax revelando un gran tumor en hemitórax derecho de 20 por $18 \mathrm{~cm}$ con efecto de masa. Exámenes destacan hipoglicemia de $24 \mathrm{mg} / \mathrm{dl} \mathrm{y} \mathrm{VHS} \mathrm{elevada.} \mathrm{Estudio}$ endocrinológico y neurológico normal, radiografías de huesos largos y manos revelan hiperostosis ósea. Se estudia con biopsia de aguja gruesa, compatible con TFSP. Se realiza resección completa por toracotomía. Biopsia definitiva confirma diagnóstico de TFSP benigno. Paciente evoluciona satisfactoriamente, resolviéndose hipoglicemia y alteraciones conductuales. Se va de alta al octavo día postoperatorio. Seguimiento a los

*Recibido el 28 octubre de 2012 y aceptado para publicación el 23 de diciembre de 2012.

Los autores no refieren conflictos de interés.

Correspondencia: Dr. Felipe León F.

Marcoleta 352, Santiago. Chile. Fax: 56-2-3543462

felipeleon81@gmail.com 
4 meses sin nuevos episodios de hipoglicemia y resolución progresiva del hipocratismo digital. Radiografías de tórax sin recidiva. Conclusiones: Los TFSP son inhabituales, se pueden presentar con algunos síndromes paraneoplásicos que deben llevar a la sospecha diagnóstica. Su tratamiento consiste en la resección completa del tumor, logrando escasa recidiva y mejoría sintomática.

Palabras clave: Tumor fibroso solitario, hipoglicemia, osteoartropatía.

\section{Introducción}

Los Tumores Fibrosos Solitarios de la Pleura (TFSP) son neoplasias infrecuentes. Se originan habitualmente de la pleura visceral y representan aproximadamente el 4 a $10 \%$ de todas las neoplasias pleurales ${ }^{1}$. Son consideradas lesiones benignas en general, pero poseen potencial maligno dependiendo de su histología ${ }^{2}$. Se manifiestan habitualmente por sintomatología respiratoria o compresiva ${ }^{3}$, asociándose en escasas ocasiones a síndromes paraneoplásicos tales como la hipoglicemia sintomática (Síndrome de Doege-Potter, SDP) ${ }^{4-6}$ y la osteoartropatía hipertrófica pulmonar (Síndrome de Pierre-MarieBamberg, SPMB) 7 .

Presentamos el caso de un paciente con un TFSP que se manifestó con ambos síndromes paraneoplásicos, los cuales revirtieron tras el tratamiento quirúrgico. Además se realiza una revisión de la literatura al respecto.

\section{Caso clínico}

Consulta en el Hospital Clínico de la Pontificia Universidad Católica de Chile un varón de 60 años previamente sano, por presentar hace 2 meses episodios reiterados de desorientación, agitación psicomotora y conductas inapropiadas (familiares refieren higiene descuidada y vestuario inadecuado). Dicho cuadro se asocia a tos productiva y baja de peso de 12 kilos en 6 meses, junto con dolor en extremidades inferiores y aumento de volumen de falanges distales de ambas manos que limitan sus actividades habituales.

Al examen físico destaca plétora facial y congestión venosa yugular, junto con disminución del murmullo pulmonar y matidez en hemitórax derecho. Además se pesquisa hipocratismo digital en ambas manos.

Se realiza radiografía de tórax (Figura 1) que revela una extensa masa lobulada que ocupa gran parte del hemitórax derecho y determina leve desplazamiento del mediastino hacia contralateral, junto a signos sugerentes de osteoartropatía hipertrófica en clavícula derecha y en segmentos visibles de diáfisis humeral izquierda, compatible con TFSP. Se complementa estudio con tomografía computada de tórax (Figura 2) confirmando un gran tumor extrapulmonar en hemitórax derecho de 22 por 18 $\mathrm{cm}$, con atelectasia pulmonar ipsilateral y desplazamiento leve del mediastino hacia contralateral. No se observan adenopatías intratorácicas ni compromiso de otras estructuras.

Los exámenes generales son normales, salvo glicemia de $24 \mathrm{mg} / \mathrm{dl}$ y VHS de 82 . Se estudia hipoglicemia con insulinemia y péptido $\mathrm{C}$ (valores disminuidos de $1,0 \mathrm{uUI} / \mathrm{ml}$ y $0,3 \mathrm{ng} / \mathrm{ml}$ respectivamente) y cortisol en valores normales. Estudio neurológico normal. Además se realizan radiografías de huesos largos y ambas manos (Figura 3) que revelan reacción perióstica laminar difusa, compatible con hiperostosis ósea secundaria.

Se maneja hipoglicemia con infusión continua de solución glucosada y se estudia tumor mediante biopsia percutánea con aguja gruesa, revelando un tumor fusocelular compatible con TFSP, estudio inmunohistoquímico positivo para CD34 y Bcl2 y negativo para EMA y actina.

Una vez descartadas otras causas de hipoglicemia se decide tratamiento quirúrgico del tumor a través de toracotomía posterolateral amplia, encontrando un gran tumor lobulado en el hemitórax derecho, sésil, el cual ingresa a mediastino posterior sin infiltrarlo. Se liberan adherencias a éstos y se logra

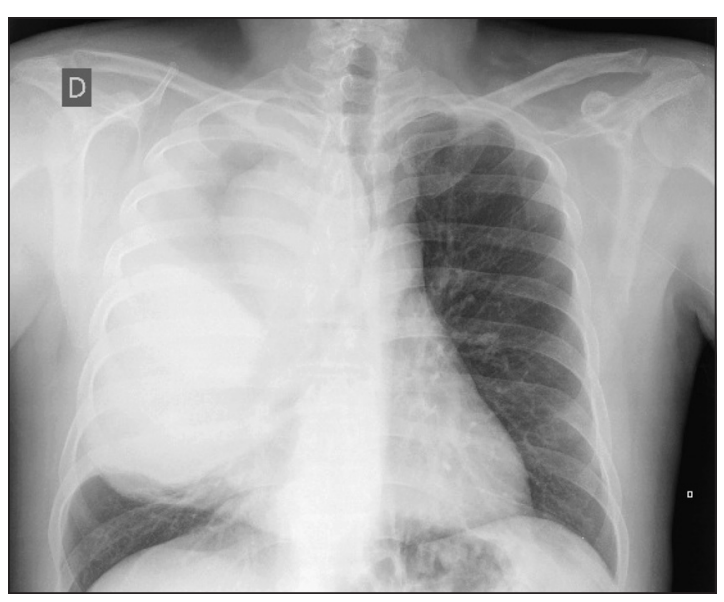

Figura 1. Radiografía de tórax posteroanterior preoperatoria: Extensa masa lobulada que ocupa gran parte del hemitórax derecho y desplaza mediastino hacia contralateral. Signos sugerentes de osteoartropatía hipertrófica en clavícula derecha y diáfisis humeral izquierda, compatible con un TFSP. 


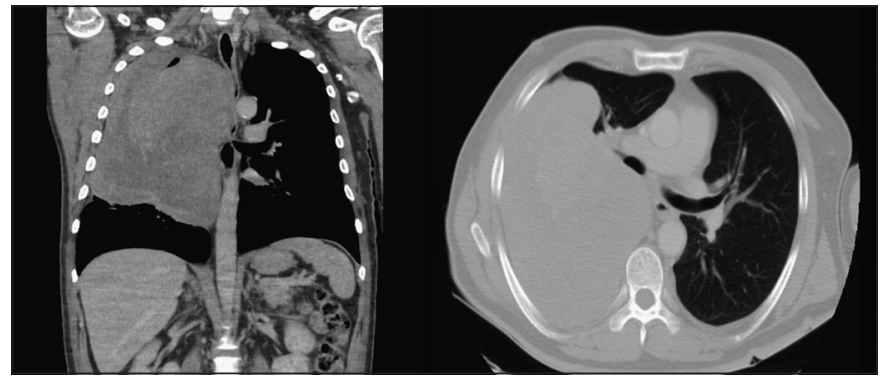

Figura 2. Tomografía de tórax preoperatoria: Gran masa extrapulmonar de base pleural que ocupa gran parte de hemitórax derecho, sin evidencias de infiltración de estructuras vecinas ni diseminación a distancia, compatible con un TFSP.

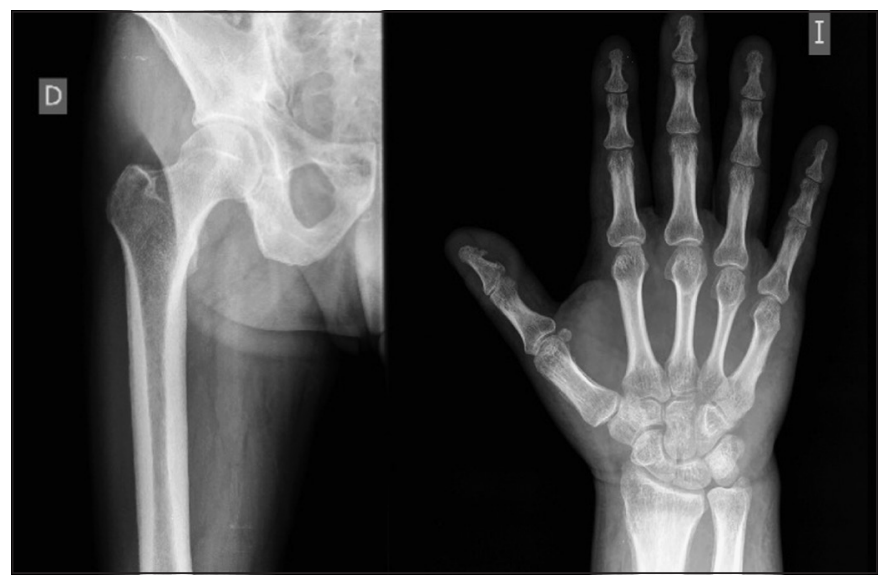

Figura 3. Radiografías de fémur derecho y mano izquierda: Reacción perióstica laminar gruesa en diáfisis de fémur y diáfisis de primer, segundo y quinto metacarpiano, así como en extremo visible de radio y cúbito, compatible con hiperostosis secundaria. extraer en su totalidad, respetando parénquima pulmonar. Se observa adecuada expansión pulmonar posterior a la exéresis tumoral.

Paciente evoluciona favorablemente, resolviéndose episodios de hipoglicemia y sin presentar nuevos episodios de alteraciones conductuales. Se retiran los drenajes pleurales al sexto día y es dado de alta al octavo día postoperatorio sin complicaciones.

La biopsia definitiva (Figura 4) reveló un TFSP polilobulado de 20 por 15 por $11 \mathrm{~cm}$ y $2.400 \mathrm{gr}$ de peso. Necrosis focal del $20 \%$ y recuento mitótico de 2 mitosis en 10 campos de aumento mayor, sin atipias celulares, confirmando benignidad de la lesión. El estudio inmunohistoquímico fue positivo para CD34 en forma difusa e intensa.

El seguimiento del paciente a los 4 meses de la cirugía sin nuevos episodios de hipoglicemia ni alteraciones conductuales. Se encuentra asintomático del punto de vista respiratorio y ha presentado resolución progresiva del hipocratismo digital y de dolor de extremidades inferiores. La radiografía de tórax al cuarto mes (Figura 5) sin evidencias de recidiva ni otras complicaciones.
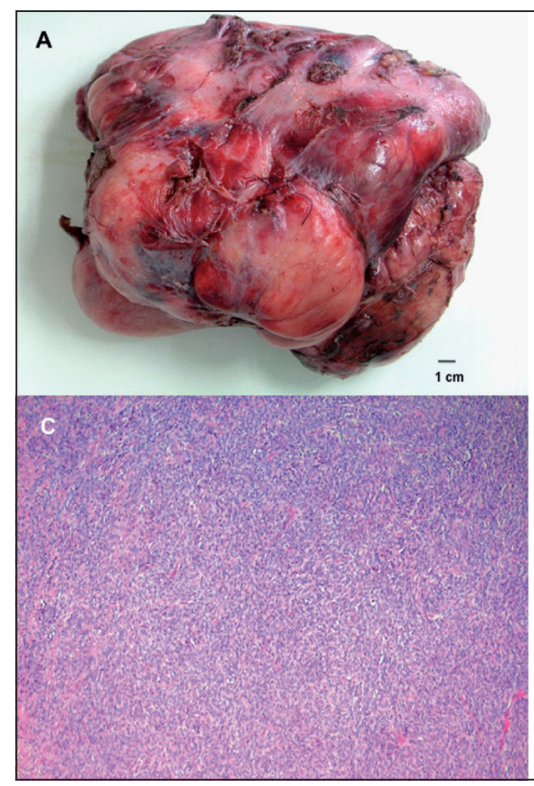

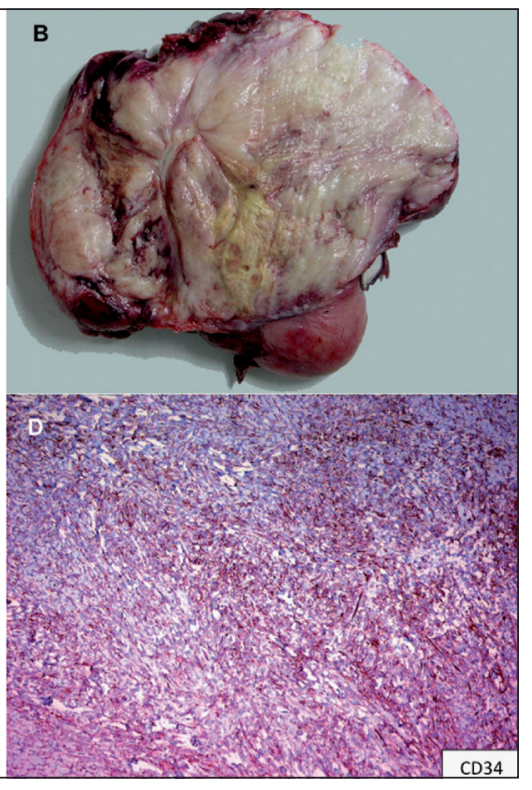

Figura 4. A. Foto de tumor resecado (cm: centímetro). B. Foto macroscópica de tumor polilobulado con área de necrosis focal del 20\%. C. Microscopía: tumor formado por células fusadas, H-E 100X. D. Inmunohistoquímica con positividad difusa e intensa para CD34. 


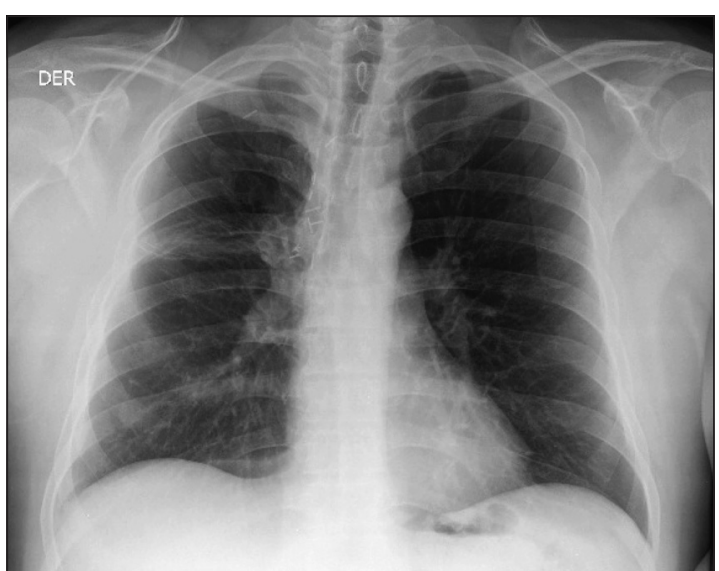

Figura 5. Radiografía de tórax posteroanterior, 4 meses postoperatoria: Sin evidencias de recidiva.

\section{Discusión}

Los TFSP fueron descritos originalmente en el año 1931 por Klemperer y Rabin ${ }^{8}$, variando su nomenclatura desde mesotelioma localizado, tumor fibroso localizado, mesotelioma fibroso y fibroma pleural. El desarrollo posterior de la microscopía electrónica e inmunohistoquímica estableció su origen en la capa submesotelial descartando un origen mesotelial ${ }^{9,10}$, permitiendo diferenciar los TFSP de los mesoteliomas.

Estos tumores son inhabituales, se han descrito aproximadamente 900 casos en la literatura ${ }^{11}$, con una incidencia de 2,8 casos por cada 100.000 hospitalizaciones ${ }^{12}$. En nuestro país, existe una importante serie publicada el año 2007 de 41 casos, en la cual se presentó hipoglicemia en $7 \%$ de los pacientes ${ }^{13}$.

Habitualmente los TFSP se presentan entre la sexta y séptima décadas de la vida, sin diferencias significativas por sexo. Además no se ha demostrado relación alguna con la exposición a asbesto, tabaco u otro agente ambiental, a diferencia del mesotelioma y otros tumores ${ }^{3}$.

La gran mayoría de estos tumores son considerados benignos. Patológicamente son neoplasias bien circunscritas, no encapsuladas, formadas por células fusadas con variabilidad en los patrones de crecimiento de las células fusiformes dentro de una misma lesión. Presentan áreas fibrosas o esclerosadas y un componente vascular prominente ${ }^{14}$. Además presentan positividad para marcadores inmunohistoquímicos como el CD34, Bcl-2, CD99 y Vimentina y negatividad para citoqueratinas, EMA, S100 y actina de músculo liso ${ }^{11,14}$.

England describió algunos criterios de malignidad tumoral, tales como una actividad mitótica mayor a 4 mitosis por 10 campos de aumento mayor, alta celularidad, presencia de necrosis o pleomorfismo nuclear ${ }^{3}$. Estas características histológicas de mal pronóstico se asocian a un peor comportamiento tumoral, con mayores índices de recurrencia tras la resección quirúrgica ${ }^{14}$.

De Perrot y cols, clasificaron morfológica y patológicamente a los TFSP en cuatro grupos bien definidos: pediculado benigno, sésil benigno, pediculado maligno y sésil maligno. Reportaron diferencias significativas en cuanto a recurrencia y sobrevida en cada uno de estos subtipos, con recurrencias de $2 \%, 8 \%, 14 \%$ y $63 \%$ respectivamente ${ }^{11}$. En nuestro caso se trató de un tumor benigno sésil, el cual presentaría buen pronóstico según esta clasificación, con escasa recidiva.

Los TFSP se pueden manifestar de diversas formas tales como disnea, dolor torácico o tos (síntomas intratorácicos), así como fiebre, baja de peso y fatiga (síntomas generales). Dichos síntomas se producen en aproximadamente el 50 a $60 \%$ de los casos, siendo este porcentaje un poco más alto en caso de tumores con características malignas $(75 \%$ aproximadamente), debido principalmente al gran tamaño que los caracteriza ${ }^{15}$.

Otras manifestaciones clínicas son su asociación a síndromes paraneoplásicos bien caracterizados, como la hipoglicemia sintomática (Síndrome de Doege-Potter) $)^{5,6}$ y la osteoartropatía hipertrófica pulmonar (Síndrome de Pierre-Marie-Bamberg).

El SDP se presenta en 2 a $4 \%$ de los TFSP ${ }^{16}$. Se ha asociado también a otros tipos de tumores intratorácicos y a algunos tumores fibrosos extratorácicos, siendo la mayoría de las veces secundario a un TFSP ${ }^{17}$. Se produce por la secreción por parte de las células tumorales de una forma incompleta del Factor de Crecimiento Insulinosímil tipo dos (IGF-II) de alto peso molecular, la cual posee la capacidad de producir los mismos efectos metabólicos que la insulina, produciendo hipoglicemia secundaria. Estudios recientes utilizando Inmunoblot han revelado niveles elevados de esta sustancia en comparación con IGF-I, IGF-II e insulina, junto con una mayor expresión de mRNA de IGF-II en pacientes con TFSP ${ }^{18,19}$. Se ha observado además una normalización de dichos valores posterior a la resección quirúrgica del tumor. Otros factores que se han asociado a este síndrome son la disminución de los mecanismos compensatorios para prevenir la hipoglicemia y también el acelerado consumo de glucosa por tumores de gran tamaño, principalmente cuando miden más de $10 \mathrm{~cm}$ como en nuestro caso ${ }^{20}$.

Respecto al SPMB, se produce en aproximadamente 10 a $20 \%$ de los TFSP. Se conoce también como osteoartropatía hipertrófica pulmonar, debido a que se produce una reacción perióstica laminar gruesa de huesos largos y manos, manifestándose 
clínicamente por "dedos en palillo de tambor" en ambas manos y dolores de extremidades. Se cree que ocurre debido a una excesiva producción de ácido hialurónico por las células tumorales, así como por efecto de la hipoxia crónica y la secreción de algunos factores de crecimiento por parte de los hepatocitos ${ }^{21}$. Estos factores serían los responsables de los cambios en el periostio observados en este síndrome ${ }^{11,14,18}$.

El tratamiento de los TFSP es la resección tumoral completa, respetando habitualmente el parénquima pulmonar. Tras la cirugía se han reportado excelentes resultados, con sobrevidas mayores al $70 \%$ a largo plazo incluso en aquellos tumores con características más agresivas ${ }^{11}$. No se ha demostrado mayor sobrevida con el uso de terapias adyuvantes, salvo cuando las resecciones han sido incompletas en las cuales podría observarse algún beneficio ${ }^{4,11}$.

Respecto a los síndromes paraneoplásicos asociados, se ha reportado en la literatura resolución completa tras la resección tumoral, tal como en nuestro caso ${ }^{18}$.

En conclusión los TFSP son tumores poco frecuentes, se manifiestan muchas veces por síntomas compresivos debido a su gran tamaño y en escasas ocasiones se asocian a síndromes paraneoplásicos tales como el SDP o el SPMB, por lo que deben sospecharse ante pacientes con hipoglicemia sin otra causa o hipocratismo digital. El tratamiento quirúrgico es efectivo en la mayoría de los casos con un bajo índice de recidiva dependiendo de la histología y características tumorales.

\section{Referencias}

1. Weynand B, Noel H, Goncetteet L, Noirhomme P, Collard P. Solitary Fibrous Tumour Of Pleura. Chest 1997;112:1424-8.

2. Hanau CA, Miettinen M. Solitary fibrous tumor: histological and immunohistochemical spectrum of benign and malignant variants presenting at different sites. Hum Pathol. 1995;26:440-9.

3. Sung SH, Chang JW, Kim J, Lee KS, Han J, Park SI. Solitary fibrous tumors of the pleura: surgical outcome and clinical course. Ann Thorac Surg. 2005;79:303-7.

4. Cardillo G, Carbone L, Carleo F, Masala N, Graziano P, Bray A, et al. Solitary fibrous tumors of the pleura: an analysis of 110 patients treated in a single institution. Ann Thorac Surg. 2009;88:1632-7.

5. Doege K. Fibrosarcoma of the mediastinum. Ann Surg. 1930;92:955-60.

6. Potter RP. Intrathoracic tumors. Radiology 1930;14:606.
7. Magdeleinat P, Alifano M, Petino A, Le Rochais J, Dulmet E, Galateau F, et al. Solitary fibrous tumors of the pleura: clinical characteristics, surgical treatment and outcome. Eur J Cardio-Thorac Surg. 2002;21:1087-93.

8. Klemperer P, Rabin LB. Primary neoplasms of the pleura: a report of five cases. Arch Pathol. 1931;11:385-412.

9. Hernandez F, Hernandez B. Localized fibrous tumors of the pleura: a light and electron microscopic study. Cancer 1974;34:1667-74.

10. Al-Azzi M, Thurlow N, Corrin B. Pleural mesothelioma of connective tissue type, localized fibrous tumor of the pleura and reactive submesothelial hyperplasia: an immunohistochemical comparison. J Pathol. 1989;158:414.

11. De Perrot M, Fischer S, Brundler M, Sekine Y, Keshavjee S. Solitary fibrous tumors of the pleura. Ann Thorac Surg. 2002;74:285-93.

12. Okike N, Bernatz PE, Woolner LB. Localized mesothelioma of the pleura. J Thorac Cardiovasc Surg. 1978;75:363-72.

13. Santolaya R, Meneses M, López J, Prats R, Fica M, González C, y cols. Tumor fibroso solitario de la pleura. Análisis de 41 casos. Rev Chil Enf Respir. 2007;23:116.

14. England DM, Hochholzer L, McCarthy MJ. Localized benign and malignant fibrous tumors of the pleura. A clinicopathologic review of 223 cases. Am J Surg Pathol. 1989;13:640-58.

15. Shields TW. Localized fibrous tumors of the pleura. En: Shields TW, ed. General Thoracic Surgery. 4th ed. Baltimore, Md: Williams \& Wilkins; 1994.

16. Zafar H, Takimoto CH, Weiss G. Doege-Potter syndrome: hypoglycemia associated with malignant solitary fibrous tumor. Med Oncol. 2003;20:403-8.

17. Herrmann BL, Saller B, Kiess W, Morgenroth K, Drochner K, Schröder T, et al. Primary malignant fibrous histiocytoma of the lung: IGF-II producing tumor induces fasting hypoglycemia. Exp Clin Endocrinol Diabetes 2000;108:515-8.

18. Kalebi A, Hale M, Wong M, Hoffman T, Murray J. Surgically cured hypoglycemia secondary to pleural solitary fibrous tumour: case report and update review on the Doege-Potter syndrome. J Cardiothorac Surg. 2009;4:45-53.

19. Frystyk J, Skjaerbaek C, Zapf J, Orskov H. Increased levels of circulating free insulin-like growth factors in patients with nonislet cell tumour hypoglycemia. Diabetologia 1998;41:589-94.

20. Fung EC, Crook MA. Doege-Potter syndrome and 'bigIGF2': a rare cause of hypoglycemia. Ann Clin Biochem. 2011;48:95-6.

21. Hojo S, Fujita J, Yamadori I, Ezaki T, Watanabe S, Yamanouchi $\mathrm{H}$, et al. Hepatocyte growth factor and digital clubbing. Intern Med. 1997;36:44-6. 